\title{
On the Construction of Trend Free Run Order of the Two-Level Factorial Design Using BIBD
}

\author{
Puja Thapliyal ${ }^{1, *}$, Veena Budhraja ${ }^{2}$ \\ ${ }^{1}$ Department of Statistics, Faculty of Mathematical Sciences, University of Delhi, Delhi, India \\ ${ }^{2}$ Department of Statistics, Sri Venkateswara College, South Campus, University of Delhi, Delhi, India
}

Email address:

pujathapliyal98@gmail.com (P. Thapliyal),veena_budhraja@gmail.com(V. Budhraja)

${ }^{*}$ Corresponding author

\section{To cite this article:}

Puja Thapliyal, Veena Budhraja. On the Construction of Trend Free Run Order of the Two-Level Factorial Design Using BIBD. American Journal of Theoretical and Applied Statistics. Vol. 9, No. 6, 2020, pp. 263-266. doi: 10.11648/j.ajtas.20200906.11

Received: September 28, 2020; Accepted: October 19, 2020; Published: October 30, 2020

\begin{abstract}
Randomization is one of the powerful tools to analyze, construct and draw valid and unbiased conclusions about the factorial design. But in some experimental situations, the technique may not perform equally well to draw valid inferences. These situations may arise due to an influence of external variations like the ageing of catalyst, known as Time-trend or Trend, on the response. Thus, instead of randomizing the run order of the factorial design, systematically arranging the order of treatments that is free of variations, neutralizes the adverse effect of Trend. Such systematic designs are known as Trend Free designs. The design gives not only higher importance to the treatments but also ensures the independence of treatments for further analysis. Methods for constructing trend-free run order of two-level designs have been studied and developed by many authors. The proposed run order for $2 \mathrm{k}$ and $2 \mathrm{k}-\mathrm{p}$ design that are linear and quadratic trend free. Systematic run order has been constructed not only to eliminate the effect of the linear and quadratic trend but also to improve design performance in the presence of a trend. This article provides another technique to develop trend free run order of two-level factorial design using Symmetric Balanced Incomplete Block Design.
\end{abstract}

Keywords: Trend Free Designs, Factorial Designs, Generalized Foldover Scheme, BIBD

\section{Introduction}

Randomizing the run order in a factorial experiment does not always give desired results. For example in a chemical experiment, as time progresses, the chemical process undergoes many chemical changes over time. The new product of the experiment may get affected by the ageing of the chemical used. In this case, the randomization technique may not give satisfactory results in the sense that the effects of interest may get affected by some external variations or trend. So, to avoid any discrepancy that arises due to the external variations, in the output, systematic pattern of level combinations of the factors or runs order with effects free of variations or trends should be constructed. Designs that generate such a run order are called Trend free designs and the run order generated is known as Trend free run order. In statistical terms, in the design, the effects are orthogonal to trend.

Cox, in ref [1] initiated the study of systematic designs.
Presence of uncontrolled variations or trend in the design led him to use the systematic technique for constructing trend free run order, instead of using the randomization technique. Other approaches to the problem of trend elimination were discussed by the author ref [2]. The author's ref [3], ref [4] and ref [5], developed plans for sequencing the treatment combinations of two-level factors to achieve an estimation of effects in the presence of a trend. The measure of uncontrolled variations in terms of the linear or quadratic trend was studied by ref [6]. Coster and Cheng as given in ref [7] introduced the generalized foldover scheme (GFS) for generating systematic run orders of any prime numbered level factorial plan from a sequence of generators in a simple way. The authors in ref [8] and ref [9] provided some new procedures for finding the optimal run order of factorial designs. In ref [10] trend free run orders of two-level designs using group theory was introduced. In ref [11] and ref [12], trend free designs were constructed using a finite field and coding theory respectively. The significant contribution to the study of two-level trend free minimum cost factorial design was 
given by the ref $[13,14]$. The statistical applications of fractional factorial designs with specific properties have been discussed in detail in Hedayat et al. as in ref [15]. In this paper, we provide a technique of generating trend free run order of two-level factorial design using Balanced incomplete block design. The paper is organized into three sections. After the introduction of the article in section 1, section 2 gives the preliminaries required throughout the article. The methodology to generate the desired designs using BIBD is described and illustrated through examples in section 3 .

\section{Preliminaries}

A factorial design with trend free run orders has many applications in industrial and manufacturing processes. Balanced Incomplete Block Designs can also be used to construct such factorial designs.

\subsection{Balanced Incomplete Block Design}

A balanced incomplete block design (BIBD) with parameters $\{\mathrm{v}, \mathrm{b}, \mathrm{r}, \mathrm{k}, \lambda\}$, is a block design with $\mathrm{v}$ treatments assigned in b blocks, each of size $\mathrm{k}$; $\mathrm{k}<\mathrm{v}$ such that each treatment is replicated $r$ times in the design and each pair of treatments occur together in $\lambda$ blocks. The parameters of BIBD satisfy the following conditions

$$
\begin{gathered}
\lambda(\mathrm{v}-1)=\mathrm{r}(\mathrm{k}-1) \\
\mathrm{rv}=\mathrm{bk} .
\end{gathered}
$$

A BIBD is symmetric if $\mathrm{b}=\mathrm{v}$ and $\mathrm{r}=\mathrm{k}$

The incidence matrix of the design is denoted by $N=\left(n_{i j}\right)_{v \times b}$ and is given as

$n_{i j}=1$ or 0 according to as $i^{\text {th }}$ treatment appearing in the $\mathrm{j}^{\text {th }}$ block or not respectively.

\subsection{Time Count}

Let $\mathrm{Y}=\left(y_{1}, y_{2}, \ldots y_{N}\right)^{\prime}$ denotes the ordered vector of observations and let $T_{x}=\left(1^{x}, 2^{x}, \ldots N^{x}\right)^{\prime}$ for $\mathrm{x}=0,1,2, \ldots \mathrm{t}$ be the $\mathrm{N} \times 1$ vector of trend coefficients and let $u_{i}$ be the contrast for the main effect $A_{i} ; \mathrm{i}=1,2, \ldots \mathrm{n}$, in the run order. Then, the quantity $u_{i}^{\prime} T_{x}$ is known as the time count for the main effect $A_{i}$.

A necessary and sufficient condition for the main effect contrast $u$ to be t-trend free is that

$$
u^{\prime} T_{x}=0 \forall x=0,1,2, \ldots, t
$$

In general, a $\mathrm{N} \times 1$ vector $u$ is called t-trend free if (1) holds.

\subsection{Trend Free Run Order}

Consider a given ordered set of treatments, represented by $\mathrm{D}_{\mathrm{N}}$ where $\mathrm{N}=2^{\mathrm{n}}$ orthogonal design of $\mathrm{n}$ factors of two levels each, are assigned to experimental units. Now, let $\mathrm{Y}=\left(\mathrm{y}_{1}, \mathrm{y}_{2}, \ldots \mathrm{y}_{\mathrm{N}}\right)$ denote the ordered vector of observations or treatments assigned. Suppose that these observations are influenced by a time trend that can be represented by a polynomial of degree $t$ $(1 \leq t \leq \mathrm{N}-1)$. Then, the model for $\mathrm{D}_{\mathrm{N}}$ can be written as

$$
Y=(X T)\left(\begin{array}{l}
\beta_{1} \\
\beta_{2}
\end{array}\right)+\mathcal{E}
$$

Where $\mathcal{E}$ is an $\mathrm{N}$-vector of uncorrelated random errors with zero means, $\mathrm{X}$ is $\mathrm{N} \times \mathrm{n}$ design matrix of factor effect coefficients. $\mathrm{T}$ is the $\mathrm{N} \times \mathbf{t}$ matrix of polynomial trend coefficients and the vector $\beta=\left(\begin{array}{l}\beta_{1} \\ \beta_{2}\end{array}\right)$ represents factor and trend parameter effects respectively.

Run order is said to be trend free of degree $t$ if it satisfies $X^{\prime} T=$ 0 where $T$ is trend vector corresponding to the values of the orthogonal polynomial of degree $t$ to $n$ equally spaced points.

\subsection{Generalized Foldover Scheme (GFS)}

Coster and Cheng as in ref [7] introduced the generalized foldover scheme (GFS) for constructing the systematic run order from the sequence of generators. A factorial design constructed by GFS has a systematic run order, which is decided by the choice and order of generators in the generator matrix. The procedure of the scheme is given as follows.

Consider a factorial design, denoted by D1. Let $U_{0}$ be a $1 \times n$ matrix of zeros. Then the run order of design D1 produced by the GFS from the generator sequence $G=$ $\left(g_{1}, g_{2}, \ldots g_{n}\right)^{\prime}$ is given by $U_{n}$ where

$$
U_{i}=U_{(i-1)}^{*}\left(g_{i}\right)=\left[\begin{array}{c}
U_{i-1} \\
U_{i-1}\left(g_{i}\right) \\
\vdots \\
U_{i-1}(q-1)\left(g_{i}\right)
\end{array}\right]
$$

For $\mathrm{i}=1,2, \ldots, \mathrm{n}$ and $\mathrm{q}$ is a prime or prime power, denoting the number of levels of the factor.

Coster and Cheng ref [7] used the Generalized Fold-over Scheme (GFS) to construct trend free designs and also discussed the conditions for linear trend free effects in GFS. They derived the following conditions for constructing $w$-trend free effects using GFS. These conditions can be used directly in the generator matrix.

1. The main effect of the given factor is $w$-trend free if the corresponding letter appears at least $(w+1)$ times in the generator sequence.

2. A two-factor interaction is w-trend free if and only if there are at-least $(\mathrm{w}+1)$ generators, in each of which exactly one of the two factors appear at a high level.

For linear trend free effect, the above conditions can be written as:

$P I$ : For any factor $X_{j}, 1 \leq j \leq n$, if there are at least two non-zero elements $x_{i j}, 1 \leq i \leq n$ then all the main effect components of factor $X_{j}$ are linear trend free.

$P I I$ : For any two factors $X_{l}$ and $X_{z}, 1 \leq l \neq z \leq n$, if there are at least two pairs $\left(x_{i l} x_{i z}\right), 1 \leq i \leq n$ such that one element is zero and the other element is non-zero. Then all components $X_{l} \times X_{Z}$ interactions are linear trend free.

Coster and Cheng in ref [7] also developed a method for the construction of generator matrices for a design. However, their method of construction is difficult to use. 


\section{3. $2^{\mathrm{n}}$ Trend Free Factorial Designs Using Symmetric BIBD}

In literature, $2^{\mathrm{n}}$ factorial Designs with trend free effects are studied and constructed by many authors using different assignment techniques as given in $\operatorname{ref}[10,11]$. In this article, we use the incidence matrix of symmetric BIBD, given in ref [16], for constructing trend free $2^{\mathrm{n}}$ Factorial Designs. The rows of incidence matrix are used as the sequence of generators. The sequence of generators then forms the generator matrix. By applying the technique of GFS to the generator matrix, the required design can be obtained. In any experimental design generally, the interest lies in the main effects and two-factor interactions only, third or higher-order interactions are usually ignored. Hence, we consider the trend free property of main effects and two-factor interactions in the generated designs. The procedure is illustrated through the following examples.

Example 1: Consider Balanced incomplete e block design with parameters $\mathrm{v}=4=\mathrm{b}, \mathrm{r}=\mathrm{k}=3, \lambda=2$. The blocks of the design are given as:

$\mathrm{d}_{1}=(124), \mathrm{d}_{2}=(123), \mathrm{d}_{3}=(134), \mathrm{d}_{4}=(234)$ and its incidence matrix is given as

$$
N=\left[\begin{array}{llll}
1 & 0 & 1 & 1 \\
1 & 1 & 0 & 1 \\
1 & 1 & 1 & 0 \\
0 & 1 & 1 & 1
\end{array}\right]
$$

The above incidence matrix of the design can be selected as a generator matrix $\mathrm{G}$ for the design as defined in (2). Applying GFS on the generator matrix $\mathrm{G}$ a $2^{4}$ factorial design is obtained. The design with all main effects and two-factor interactions is given in Table 1. The last column in the table is the $\mathrm{N}$ 1vector of polynomial trend coefficients. The last row of the table gives the time count for the effects. Time count is calculated by replacing zeroes by -1 in the design matrix and obtaining the sumproduct of each column with the last column of the table. We observe here that the time count for all the effects is zero that means, the effects are at-least linear trend free. The result can be obtained using the properties given in sub-section 2.4. We observe here that each column of the generator matrix labelled as $\mathrm{X}_{1}, \mathrm{X}_{2}, \mathrm{X}_{3}$ and $\mathrm{X}_{4}$ has at-least two non-zero elements and for two-factor interactions, $X_{i} X_{j}(i \neq j, i$, $j=1,2,3,4)$ there exist two pairs in which one element is zero and the other is non-zero. So, by the properties PI and PII, the generated design has all main effects and two factors interactions linear trend free or trend free of degree one.

Table 1. Linear trend free $2^{4}$ factorial design.

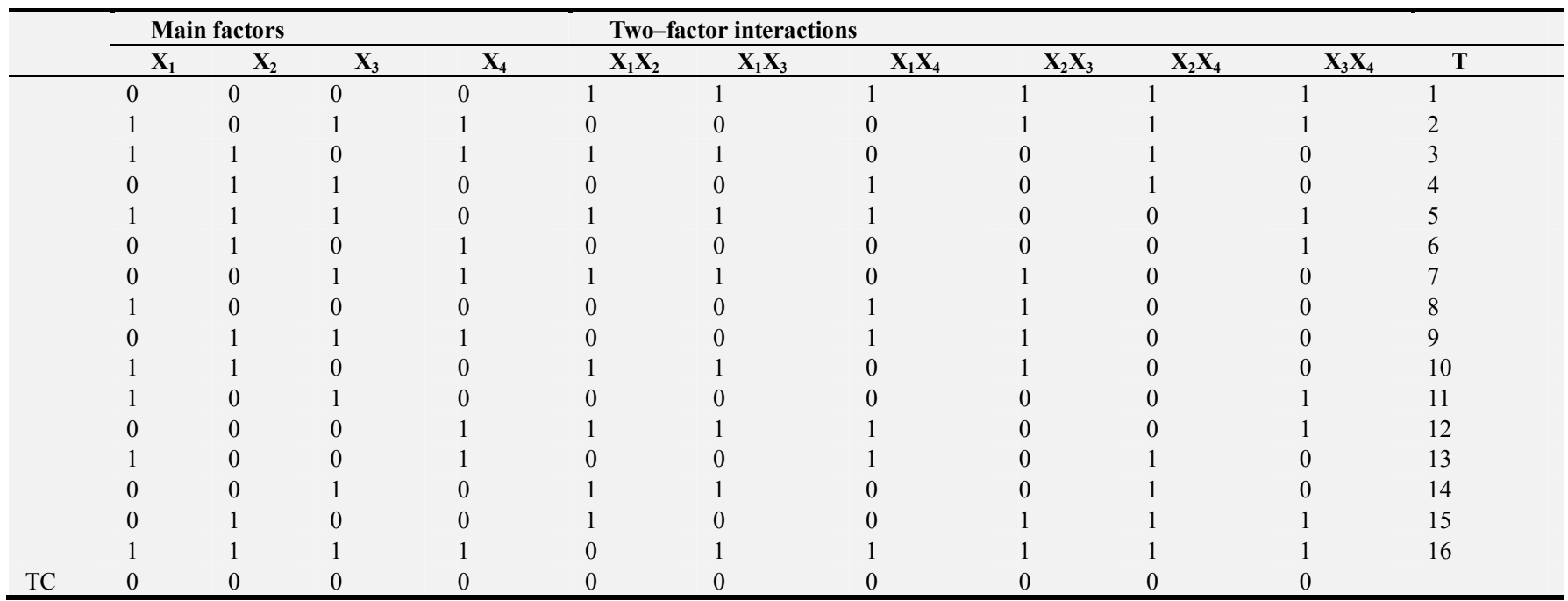

Example 2: Consider Balanced incomplete Block Design with parameters $\mathrm{v}=7=\mathrm{b}, \mathrm{r}=3=\mathrm{k}, \lambda=1$

The blocks of the design is given as: $d_{1}=\{0,1,3\}, d_{2}=\{1,2,4\}$,

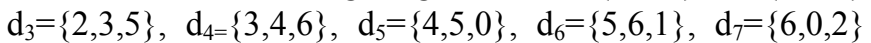
and its incidence matrix $\mathrm{N}$ is given as

$$
\mathrm{N}=\left[\begin{array}{lllllll}
1 & 0 & 0 & 0 & 1 & 0 & 1 \\
1 & 1 & 0 & 0 & 0 & 1 & 0 \\
0 & 1 & 1 & 0 & 0 & 0 & 1 \\
1 & 0 & 1 & 1 & 0 & 0 & 0 \\
0 & 1 & 0 & 1 & 1 & 0 & 0 \\
0 & 0 & 1 & 0 & 1 & 1 & 0 \\
0 & 0 & 0 & 1 & 0 & 1 & 1
\end{array}\right]
$$

The columns of the matrix are labelled as $\left.X_{i,} \mathrm{i}=1,2 \ldots, 7\right)$.
Applying GFS in the incidence matrix we get a $2^{7}$ factorial design. Using PI, all the main effects are quadratic trend free and according to PII, all two-factor interactions are 4-trend free.

The above results can be generalized and expressed in the following theorems:

Theorem 1: If there exists a symmetric BIBD with parameters $\{\mathrm{v}, \mathrm{b}, \mathrm{r}, \mathrm{k}, \lambda\}, \mathrm{r}, \mathrm{k} \geq 2$, then a $2^{\mathrm{v}}$ factorial design can be constructed such that every main effect is (k-1)-trend free.

Proof: Consider the incidence matrix of $\operatorname{BIBD}(\mathrm{v}, \mathrm{b}, \mathrm{r}, \mathrm{k}, \lambda)$. Since the block size of each block is $\mathrm{k}$, hence every column of incidence matrix will have at most $\mathrm{k}$ non-zero elements. Applying GFS to the incidence matrix a $2^{\mathrm{v}}$ factorial design is 
constructed and using the conditions given by ref [7] all the main effects are (k-1) trend free.

Theorem 2: Existence of a symmetric BIBD with parameters $\{\mathrm{v}, \mathrm{b}, \mathrm{r}, \mathrm{k}, \lambda\}, \mathrm{r}, \mathrm{k} \geq 2$, implies the existence of a $2^{\mathrm{v}}$ factorial design in which all two-factor interactions are 2 $(\mathrm{r}-\lambda)-1$ trend free.

Proof: Consider a BIBD (v, b, r, k, $\lambda$ ). Let a treatment $\alpha_{\mathrm{I}}$ appears in $r$ blocks. This implies that it does not appear in (b-r) blocks. Now consider two treatments $\alpha_{\mathrm{I}}$ and $\alpha_{\mathrm{j}}$ and suppose they occur together in $\lambda$ blocks. Also, $(\mathrm{r}-\lambda)$ blocks contain $\alpha_{\mathrm{I}}$ but not $\alpha_{\mathrm{j}}$, and (r- $\lambda$ ) blocks contain $\alpha_{\mathrm{j}}$ but not $\alpha_{\mathrm{i} \text {. So the number }}$ of blocks which contain neither $\alpha_{\mathrm{I}}$ nor $\alpha_{\mathrm{j}}$ is $\mathrm{b}-2 \mathrm{r}+\lambda$. This gives that any two columns of the incidence matrix will have at-least $2(\mathrm{r}-\lambda)$ generators in each of which exactly one of the two factors appear at a high level. Using the incidence matrix as the generator matrix and applying GFS we get a $2^{\mathrm{v}}$ factorial design in which all two-factor interactions are $2(\mathrm{r}-\lambda)-1$ trend free.

In Table 2 we have listed some trend free $2^{\mathrm{v}}$ factorial designs generated from a series of symmetric BIBD as in ref [16] using the above results.

Table 2. List of Generated Designs.

\begin{tabular}{|c|c|c|c|}
\hline \multicolumn{4}{|c|}{ 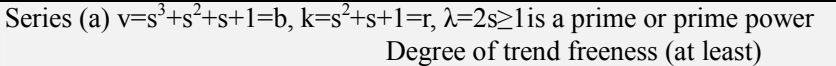 } \\
\hline & Factorial Design & Main effects & Factor Interactions \\
\hline & $2^{s^{3}+s^{2}+s+1}$ & $s^{2}+s$ & $2\left(s^{2}+s-1\right)-1$ \\
\hline$s=1$ & $2^{4}$ & Quadratic & linear \\
\hline$s=2$ & $2^{15}$ & 6-trendfree & 9-trendfree \\
\hline$s=3$ & $2^{40}$ & 12-trendfree & 21-trendfree \\
\hline \multicolumn{4}{|c|}{ Series (b): $\mathrm{v}=2^{\mathrm{s}}-1=\mathrm{b}, \mathrm{r}=2^{\mathrm{s}-1}-1=\mathrm{k}, \lambda=2^{\mathrm{s}-2}-1 ; \mathrm{s}>2$} \\
\hline & $2^{2^{s}-1}$ & $\left(2^{\mathrm{s}-1}-2\right)$-trend free & $\left(2^{s-1}-1\right)$-trendfree \\
\hline$s=3$ & $2^{7}$ & 2-trendfree & 3-trendfree \\
\hline$s=4$ & $2^{15}$ & 6 -trendfree & 7-trendfree \\
\hline$s=5$ & $2^{31}$ & 14-trendfree & 15-trendfree \\
\hline \multicolumn{4}{|c|}{ Series (c): $v=s^{2}+s+1=b, r=s+1=k, \lambda=1$} \\
\hline & $2^{s^{2}+s+1}$ & s-trend free & $2 \mathrm{~s}-1$ trendfree \\
\hline$s=2$ & $2^{7}$ & 2-trendfree & 3-trendfree \\
\hline$s=3$ & $2^{13}$ & 3-trendfree & 5-trendfree \\
\hline$s=4$ & $2^{21}$ & 4-trendfree & 7-trendfree \\
\hline$s=5$ & $2^{31}$ & 5-trendfree & 9-trendfree \\
\hline
\end{tabular}

\section{Conclusion}

Trend free two-level factorial designs were also constructed using coding theory in which the trend free property was confined to main effects only. Factorial designs with trend free run orders have immense applications in many industrial, manufacturing, and agricultural units. The main factors of a design are always being a prior interest to a manufacturer. Sometimes, the effect of interactions of factors on the response may prove to be significant to get the optimal run order of a factorial design. This article provides an easy and general procedure to generate $2^{\mathrm{n}}$ factorial design in which the main factor and two-factor interactions are at-least linear trend free.

\section{Acknowledgements}

The research was funded by the Department of Science and
Technology, India grant SR/WOS-A/PM-13.

\section{References}

[1] D. R. Cox, "Some Systematic Experimental Designs," Biometrika, Vol. 38, 1951, pp. 312-323.

[2] G. E. P. Box, "Multi-factor Designs of First Order," Biometrika, Vol. 39, 1952, pp. 49-57.

[3] M. Jacroux, "On the Construction of Trend Resistant Mixed Level Factorial Run Orders,” Annals of Statistics, Vol. 22, 1994, pp. 904-916.

[4] G. E. P. Box, and W. A. Hay, "Statistical design for the Efficient Removal of Trend so occurring in a Comparative Experiment with an Application in Biological Assay," Biometrics, Vol. 9, 1953, pp. 304-319.

[5] H. H. Hill, "Experimental Designs to adjust for time trends," Technometrics, Vol. 2, 1960. pp. 67-82.

[6] C. Daniel, and F. Wilcoxon, "Factorial 2p-q plans robust against Linear and Quadratic Trends," Technometrics. Vol. 8, 1966, pp. 259-278.

[7] D. C. Coster, and C. S. Cheng, "Minimum Cost Trend Free Run Orders of Fractional Factorial Design," The Annals of Statistics, Vol. 16 (3), 1988, pp. 1188-1205.

[8] C. S. Cheng, "Run Orders of Factorial Designs," Proceedings of the Berkeley Conference in Honor of Jerzy Neyman and Jack Kiefer, Lucein. Vol. 2, 1985, pp. 619-633.

[9] C. S. Cheng, "Construction of Run Orders of Factorial Design," Statistical Design and Analysis of Industrial Experiment, Marcel Dekkar, New York. 1990, pp. 423-439.

[10] C. S. Cheng, and M. Jacroux, "On the Construction of Trend Free Run Order of Two-Level Factorial Designs," American Statistical Association, Vol. 83, 1988, pp. 1152-1157.

[11] P. Singh, P. Thapliyal, and V. Budhraja, "Construction of Fractional Factorial Designs with some Linear Trend Free Effects through Finite Field," Journal of combinatorics, Information and System Sciences, Vol. 39 (1-2), 2014, pp. $57-76$.

[12] P. Singh, P. Thapliyal, and V. Budhraja, "Construction of Linear Trend-free fractional factorial designs using linear codes," International journal of agricultural and statistical sciences, Vol. 12 (1), 2016, pp. 13-19.

[13] R. W. Mee, and A. V. Ramanova,). "Constructing and Analyzing two-level ltrend robust designs," Quality Engineering, Vol. 22 (4), 2010, pp. 306-316.

[14] H. Hilow, "Minimum cost linear trend free fractional factorial designs," Journal of statistical theory and practice Vol. 6, 2012, pp. 580-589.

[15] A. S. Hedayat, N. J. A, Sloane, and J. Stufken. Orthogonal arrays; Theory and Applications, Springer-Verlag, New York. 1999.

[16] C. R. Rao, "Some Combinatorial Problems of Arrays and Applications to Design of Experiments," A Survey of Combinatorial Theory, in N. Srivastava ed., Amsterdam, North-Holland. 1973, pp. 349-359. 